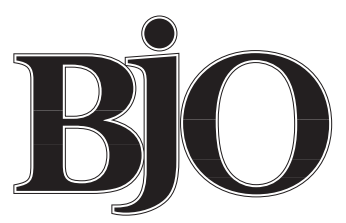

British Journal of Ophthalmology

In this issue of the $B F O$ ( $\mathrm{p} \mathrm{6)}$, Gandorfer and colleagues contribute to the recent burgeoning of research on enzymatic vitreolysis. Vitreoretinal surgeons, the beneficiaries in recent years of many surgical advances-silicone oil and long acting gas tamponade, perfluorocarbon liquids, photodynamic therapy - nevertheless harbour a collective "wish list" of surgical agents and devices awaiting development. Such a list would include long term heavy liquid tamponade, retinal adhesives, antifibroproliferative drugs, and antiangiogenic treatments. But none figures so prominently as enzymatic vitreolysis, which for years has held the promise of minimally invasive solutions for surgical problems in vitreoretinal diseases such as diabetic retinopathy and macular hole.

The goal is to develop chemicals which when injected into the vitreous produce selective vitreolysis, in the form of either posterior vitreous detachment (PVD) or vitreous liquefaction. Enzymatic vitreolysis is envisaged to augment or even replace standard mechanical vitrectomy, over which it presents important advantages. It offers lower operative risks, less surgeon time, lower costs, greater patient access, and a transition to office based vitreoretinal procedures.

A number of vitreolytic substances have been investigated, including hyaluronidase, dispase, tissue plasminogen activator, and chondroitinase. Plasmin has perhaps received the most attention; it is a non-specific protease with action against components of the vitreoretinal interface-namely, laminin and fibronectin. By degrading the links between the cortical vitreous and the internal limiting membrane (ILM), it becomes possible to produce therapeutic PVD. Gandorfer and colleagues demonstrate ultrastructurally that it is indeed possible to use intravitreal injection of plasmin to create a complete PVD. In controlled experiments in postmortem pig eyes, light and scanning electron microscopy verified that at sufficient concentrations and incubation times, plasmin injected eyes showed PVD with the retinal surface smooth and free of cortical vitreous remnants. It is significant that enzymatic action alone was sufficient to induce PVD without adjuvant gas bubble injection or cryotherapy necessitated in other studies. ${ }^{1-3}$

The full realisation of an effective agent for enzymatic vitrectomy holds the promise of creating a raft of new therapeutic strategies for vitreoretinal disease. Macular hole repair offers a good example; in conventional surgery removal of the posterior cortical vitreous is considered critical to relieve tractional forces on the macular hole. ${ }^{4}$ However, mechanically peeling the hyaloid from the retinal surface can be technically difficult, is associated with the risk of retinal breaks, and has even been implicated in postoperative visual field loss. ${ }^{5}$ Trese and colleagues ${ }^{6}$ have recently presented results of plasmin assisted vitrectomy in patients with stage 3 macular hole; injection of autologous plasmin before standard vitrectomy was reported to achieve satisfactory PVD and facilitate surgical repair of the hole. Furthermore, the procedure has shown promise in the more challenging cases of macular holes caused by ocular trauma. ${ }^{7}$ It becomes conceivable that an office based procedure utilising vitreolytic PVD, perhaps with injection of expansile gas tamponade, could in some cases obviate standard vitrectomy in macular hole repair.

In diabetic vitreous haemorrhage, enzymatic vitreolysis is being investigated as a means of accelerating visual resolution as well as allowing earlier application of panretinal photocoagulation. Enzymatic vitrectomy could eventually prove useful in diabetic tractional retinal detachment (TRD), as an adjunct to surgery to relieve vitreoretinal traction. Furthermore, it offers a means to induce PVD in patients with proliferative diabetic retinopathy at risk for developing TRD. Enzymatic PVD could neutralise the ability of the vitreous to act as a scaffolding for neovascular ingrowth and subsequent traction retinal detachment. Could enzymatic PVD become a prophylactic intervention for diabetic patients in the early stages of proliferative or even non-proliferative retinopathy as a way of pre-empting vision threatening complications?

Other applications can be envisaged. Enzymatic vitreolysis might be useful as a supplement to pneumatic retinopexy for rhegmatogenous retinal detachment repair; injecting an enzyme at the time of gas injection to additionally release vitreous traction could potentially increase the success rate of this office based procedure. As another example patient complaints associated with vitreous floaters are all too familiar to ophthalmologists, but the risk-benefit profile for surgery for vitreous floaters is prohibitive. Could enzymatic vitreolysis reduce risks sufficiently to become viable for the large number of patients with this relatively benign but annoying visual problem? The arrival of enzymatic vitreolysis may expand vitreoretinal practice in ways that can't be predicted.

Before enzymatic vitreolysis enters the mainstream central questions will need to be addressed-effectiveness, inflammatory responses, retinal toxicity, long term complications. And years of work in the field of vitreolytic 
enzymes have yet to yield a widely accepted alternative to mechanical vitrectomy. Nevertheless, as the limits of conventional vitrectomy are being approached vitreoretinal surgeons continue to look forward over the next years to a new generation of therapies with vitreolytic enzymes.

ROBERT B BHISITKUL

Department of Ophthalmology, University of California San Francisco, 10 Kirkham Street, K301, San Francisco, CA 94143, USA

bhisit@itsa.ucsf.edu
1 Kang SW, Hyung SM, Choi MY, et al. Induction of vitreolysis and detachment with hyaluronidase and perfluoropropane gas. Kor F Ophthalmol 1995;9:69-78. with hyaluronidase and perfluoropropane gas. Kor f Ophthalmol 1995;9:69-78.
Hikichi T, Yanagiva N, Kado M. Posterior vitreous detachment induced by

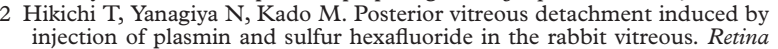
injection of plasmin and sulfur hexafluoride in the rabbit vitreous. Retina
1999;19:55-8. 3 Hesse L, Nebeling B, Schroeder B, Heller G, et al. Induction of posterior vitreous detachment in rabbits by intravitreal injection of tissue plasminogen activator following cryopexy. Exp Eye Res 2000;70:31-9.

4 Kelly NE, Wendel RT. Vitreous surgery for idiopathic macular holes. Results of a pilot study. Arch Ophthalmol 1991;109:654-9.

5 Cullinane AB, Cleary PE. Prevention of visual field defects after macular hole surgery. Br F Ophthalmol 2000;84:372-7.

6 Trese MT, Williams GA, Hartzer MK. A new approach to stage 3 macular holes. Ophthalmology 2000;107:1607-11.

7 Chow DR, Williams GA, Trese MT, et al. Successful closure of traumatic macular holes. Retina 1999;19:405-9.

\section{Adjustable suture strabismus surgery: continuing progress}

The use of the adjustable suture strabismus technique has led to a revolution in strabismus surgery. Previously, depending on the surgeon's experience, the results of strabismus surgery may not have been very predictable after a first eye muscle procedure, let alone subsequent ones. The advent of adjustable sutures permitted ophthalmologists to adopt new attitudes towards their patients. Firstly, non-experienced ophthalmologists could attempt surgery, knowing that if their approach were not quite right, they would be given a second chance during the postoperative adjustment process. Secondly, experienced strabismus surgeons could realistically foretell a successful outcome rate often exceeding $90 \%$ - a prediction practically impossible without the use of adjustable sutures.

As with any innovative procedure, surgeons then attempted to improve the technique. The paper in this issue of the $B \mathcal{F O}$ ( $\mathrm{p} \mathrm{80)}$ ) by Choi and colleagues is such a potential improvement. Their premise is that the further in time from the actual strabismus surgery the adjustable process is performed, the higher the success rate should be. Delaying the adjustment as long as 6 or more days after the surgery has been reported, but not often practised. ${ }^{1}$ This premise assumes that in the immediate postoperative period a number of factors may still change, which would affect the ultimate ocular alignment. Among these factors may be further healing of the muscle and conjunctiva, oedema of adjacent tissues especially Tenon's capsule, ability of the patient to properly focus on the fixation target, and changes in the length-tension curve of the operated muscle(s). Despite these concerns, Ruben and Elston, and Biglan and associates have reported good results when adjustment is performed within minutes of completing the initial surgery, often with the patient still on the operating table. ${ }^{23}$ These authors would not find ADCON-L useful because they used early adjustment. Even for those authors who perform the adjustment process later than the immediate postoperative period, there has been little difficulty with adjustment. Spierer and, later, Velez and associates reported adjustment at about 24 hours postoperatively to be essentially as easy as at 6 hours with similar long term results. ${ }^{45}$ Thus, the approach of Choi and colleagues might be most useful for adjustments performed beyond 24 hours after initial surgery.

The ADCON-L apparently works by preventing adhesion between tendon and sclera. This is thought to be accomplished by blocking fibroblast migration, either chemically or mechanically. In a multicentre controlled trial in 298 patients undergoing lumbar discectomy, ADCON-L significantly reduced peridural scar formation, postoperative related pain, low back pain, and permitted more movement of extremities. ${ }^{6}$ After lumbosacral discectomy, epidural scar tissue was found to be absent or minimal. ${ }^{7}$ Other agents have been placed on the sclera for the same purpose but have not found common use. Some of them, such as mitomycin-C and 5-fluorouracil, are potentially toxic to the eye, whereas ADCON-L appears to be non-toxic. ${ }^{89}$

Another question to raise about this use is if the delayed healing imposed by ADCON-L, at least in the rabbit model, will leave the eventual adhesion between tendon and sclera weaker than it would otherwise be. If so, the impaired adhesion could further weaken the effects of a muscle recession or resection. In addition, the muscle could more easily dehisce off the globe after trauma. The authors' finding that the disinsertional force 3 weeks after surgery was no different whether an eye had been treated with ADCON-L or balanced salt solution is encouraging. But their other finding that even at 3 weeks postoperatively, the eyes treated with ADCON-L revealed less fibrosis on histological examination is worrisome with regard to the structural integrity of the scleral adhesion.

If the adjustment will be delayed more than 24 hours after surgery, another consideration is patient convenience. With one or more long sutures in the conjunctival sac, the patient will probably be patched, which would preclude much social interaction. In addition, the long sutures would cause discomfort in many patients. Perhaps these negative aspects of deferred adjustment could be justified if the long term result from a deferred adjustment could be proved to be significantly better than adjustment within 24 hours of surgery.

The results of Choi and associates are stimulating. A human trial of ADCON-L and performing the adjustment more than 24 hours after initial surgery should address a number of questions. In the human, will ADCON-L prove as effective and non-toxic as shown in the rabbit model? Does the deferred adjustment improve long term results? Is the discomfort of maintaining long sutures in the eye for a prolonged period tolerable to the patient? Will a muscle adjusted after use of ADCON-L maintain a weakened attachment to the sclera? These initial results in a rabbit model are sufficiently convincing and exciting to justify a human trial to answer these questions.

SHERWIN J ISENBERG

Jules Stein Eye Institute, Department of Ophthalmology, UCLA School of Medicine, 100 Stein Plaza, Los Angeles, CA 90095-7001, USA

isenberg@ucla.edu 
1 Howard CW, Smith AG. Use of adjustable sutures: a helpful modification. Ann Ophthalmol 1986;18:70-3.

2 Ruben ST, Elston JS. One stage adjustable sutures: practical aspects. $\mathrm{Br} \mathcal{F}$ Ophthalmol 1992;76:675-7.

3 Biglan AW, Davis JS, Day R, et al. Prospective investigation of the effectiveness of intraoperative adjustable sutures for correction of strabismus. Trans Am Ophthalmol Soc 1994;92:325-47.

4 Spierer A. Adjustment of sutures 8 hours vs 24 hours after strabismus surgery. Am f Ophthalmol 2000;129:521-4.

5 Velez FG, Chan TK, Vives T, et al. Timing of postoperative adjustment in adjustable suture strabismus surgery. 7 Am Ass Pediatr Ophthalmol Strabismus (in press).
6 De Tribolet N, Pochet F, Lutz TW, et al. Clinical assessment of a novel antiadhesion barrier gel: prospective, randomized, multicenter, clinical trial of ADCON-L to inhibit postoperative peridural fibrosis and related symptoms after lumbar discectomy. Am f Orthop 1998;27:111-20.

7 Brotchi J, Pirotte B, De Witte O, et al. Prevention of epidural fibrosis in a prospective series of 100 primary lumbo-sacral discectomy patients: follow-up and assessment at re-operation. Neurol Res 1999;21(Suppl):S4750.

8 Cruz OA. Evaluation of mitomycin to limit postoperative adhesions in strabismus surgery. F Pediatr Ophthalmol Strabismus 1996;33:89-92.

9 Hwang J-M, Chang BL. Combined effect of Interceed and 5-fluorouracil on delayed adjustable strabismus surgery. Br f Ophthalmol 1999;83:788-91.

\section{Video Reports (www.bjophthalmol.com)}

Capsule staining and mature cataracts: a comparison of indocyanine green and trypan blue dyes D F Chang Pearls for implanting the Staar toric IOL D F Chang 